\title{
Hippocampal Plasticity across Multiple Days of Exposure to Novel Environments
}

\author{
Loren M. Frank, ${ }^{1,2,3,4}$ Garrett B. Stanley, ${ }^{2,3}$ and Emery N. Brown ${ }^{1,3}$ \\ ${ }^{1}$ Neuroscience Statistics Research Laboratory, Department of Anesthesia and Critical Care, Massachusetts General Hospital, Boston, Massachusetts 02114, \\ ${ }^{2}$ Division of Engineering and Applied Sciences, Harvard University, Cambridge, Massachusetts 02138, ${ }^{3}$ Harvard/Massachusetts Institute of Technology, \\ Division of Health Sciences and Technology, Cambridge, Massachusetts 02139, and ${ }^{4}$ Keck Center for Integrative Neuroscience, Department of Physiology, \\ University of California San Francisco, San Francisco, California 94143
}

The hippocampus is essential for learning complex spatial relationships, but little is known about how hippocampal neural activity changes as animals learn about a novel environment. We studied the formation of new place representations in rats by examining the changes in place-specific firing of neurons in the CA1 region of the hippocampus and the relationship between these changes and behavioral change across multiple days of exposure to novel places. We found that many neurons showed very rapid changes on the first day of exposure to the novel place, including many cases in which a previously silent neuron developed a place field over the course of a single pass through the environment. Across the population, the largest changes in neural activity occurred on day 2 of exposure to a novel place, but only if the animal had little experience $(<4 \mathrm{~min})$ in that location on day 1 . Longer exposures on day 1 were associated with smaller changes on day 2, suggesting that hippocampal neurons required 5- 6 min of experience to form a stable spatial representation. Even after the representation stabilized, the animals' behavior remained different in the novel places, suggesting that other brain regions continued to distinguish novel from familiar locations. These results show that the hippocampus can form new spatial representations quickly but that stable hippocampal representations are not sufficient for a place to be treated as familiar.

Key words: hippocampus; learning; memory; spatial; adaptive estimation; place cells

\section{Introduction}

The hippocampal system is essential for learning complex relationships (O'Keefe and Nadel, 1978; Squire and Zola-Morgan, 1991; Eichenbaum and Cohen, 2001; Holscher, 2003), and both hippocampal neural activity and hippocampal synaptic plasticity are crucial for learning spatial tasks (Pacteau et al., 1989; Morris et al., 1990; Jarrard, 1993; Moser et al., 1993; Izquierdo et al., 1997; Cassel et al., 1998; Ferbinteanu et al., 2003). In addition, rat hippocampal neurons fire in a place-specific manner as an animal explores a familiar environment (O’Keefe and Dostrovsky, 1971; Muller, 1996; Best et al., 2001). These place cells are thought to play an important role in spatial navigation and spatial memory in the rat, and place- and view-specific hippocampal activity has been reported in both humans and non-human primates (Rolls, 1999; Ekstrom et al., 2003; Hori et al., 2003).

Received May 20, 2004; revised July 15, 2004; accepted July 23, 2004.

This work was supported by National Institutes of Health (NIH) Grant MH65108 to L.M.F., Whitaker Foundation Grant RG-01-0087 to G.B.S., and NIH Grants MH59733, MH61673, and DA015644 to E.N.B. We thank Kyoko Kaneda and Timothy Warren for help training animals and building the experimental apparatus and Mark Baxter for help with the histology. We also thank Riccardo Barbieri, Uri Eden, Anita Frank, Ana Nathe, Victor Solo, Michael Stryker, and Wendy Suzuki for helpful comments on the analyses and this manuscript.

Correspondence should be addressed to either of the following: Dr. Loren M. Frank, Keck Center for Integrative Neuroscience, Department of Physiology, University of California San Francisco, S-762, 513 Parnassus Avenue, San Francisco, CA 94143, E-mail: Ioren@phy.ucsf.edu; or Dr. Emery N. Brown, Neuroscience Statistics Research Laboratory, Department of Anesthesia and Critical Care, Massachusetts General Hospital, Clinics 3, 55 Fruit Street, Boston, MA 02114, E-mail: brown@neurostat.mgh.harvard.edu.

DOI:10.1523/JNEUROSCI.1958-04.2004

Copyright $\odot 2004$ Society for Neuroscience $\quad 0270-6474 / 04 / 247681-09 \$ 15.00 / 0$
To date, most work on the hippocampus has examined place cell activity in environments in which the animal had substantial experience before neural activity was recorded (Muller, 1996). As a consequence, relatively little is known about the changes in hippocampal activity that occurs during the initial exploration of a novel environment. Those studies that examined activity during novel exposures have suggested that hippocampal spatial responses or place fields can often be seen on the very first traversal of a new place (Hill, 1978) and that hippocampal population activity accurately represents the position of the animal once it has had $\sim 10$ min of experience in the new place (Wilson and McNaughton, 1993). Studies that examined the effects of environmental or task-related manipulations on place field structure reported that alterations in visual cues, environmental boundaries, or behavioral task can cause large changes in hippocampal place fields (Bostock et al., 1991; Markus et al., 1995; Fyhn et al., 2002; Knierim, 2002; Lever et al., 2002). These changes were identified by averaging neural spiking activity across several minutes of experience, so the moment-by-moment dynamics of these spatial representations have not been identified. In addition, the changes in neural activity across multiple days of exposure to a new place and their relationship to behavior is not yet understood, in part because of the difficulty of accurately quantifying rapid changes in place field structure.

We recently developed an adaptive point process estimation algorithm to track changes in receptive field structure on a millisecond time scale (Brown et al., 2001; Frank et al., 2002; Eden et 
al., 2004). This algorithm makes it possible to quantify rapid changes in spatial representations. Here, we recorded from hippocampal neurons while animals explored novel and familiar places and used this algorithm to examine the characteristics of place field plasticity across multiple days of exposure to a new place. We also related changes in spatial activity to changes in behavior related to the animal's familiarity with the environment.

\section{Materials and Methods}

Subjects and behavioral training. Four male Long-Evans rats were handled, food deprived to $85 \%$ of their baseline weight, and trained to run for Yoohoo chocolate drink reward in a continuous alternation task on a T-maze. The T-maze consisted of three arms of a $152 \mathrm{~cm}$ diameter eightarm maze (see Fig. $1 A$ ). During training, animals only had access to these three arms. The apparatus was located in a room with a number of prominent visual cues including the data collection system, doors, and lights. In this task, each animal was initially placed in the home arm (arm 1) and was rewarded for running in the following pattern: 1-3-1-7-1-3$1-\ldots$. The animal was rewarded each time it reached the end of the next correct arm. If the animal selected the wrong outside arm on an outbound trip (e.g., arm 7 instead of arm 3), it was only rewarded if it went back to the home arm and then to arm 3 . This task is a continuous version of a T-maze alternation task, the correct performance of which is impaired by hippocampal damage (Rawlins and Olton, 1982; Aggleton et al., 1986), but the effect of hippocampal lesions on this particular task has not yet been established. Animals were given one or two 20 min sessions per day. Once the animals were performing at $80 \%$ correct, in which a single correct trial involved a run from the home arm to the correct outside arm ( $\operatorname{arm} 3$ or arm 7) and back to the home arm, we implanted a 30 tetrode microdrive array. A total of 8 tetrodes targeted the CA1 region of the hippocampus (anteroposterior, -3.6; lateral, 2.2), and 22 tetrodes targeted the entorhinal and perirhinal cortices (anteroposterior, - 7.4; lateral, 4.0; angled outward from the midline at $10^{\circ}$ ). All surgical procedures and postsurgical care were performed according to Harvard University and National Institutes of Health guidelines. At the end of data collection, we perfused the animals and verified the locations of electrodes. The specific surgical and histological methods have been described previously (Frank et al., 2000). Here, we focus on changes in CA1 neural activity.

Data collection. After the animals had recovered from surgery, we began recordings. Each day of recording consisted of five epochs: (1) a 20-30 min rest period in which the animal sat on a platform in a highwalled box; (2) a 20 min run in the familiar configuration; (3) a 20-30 min rest period; (4) a 20-25 min run in the novel configuration; and (5) a final 20-30 min rest period. The run in the novel configuration was ended when the animal became satiated or when $25 \mathrm{~min}$ had elapsed. In the novel configuration, either arm 3 or arm 7 was blocked off, a novel arm was opened, and the animal had to learn to run in the corresponding pattern (e.g., 1-3-1-6-1-3-1-... ) (see Fig. 1A). The novel arm was always on the same side of the home arm as the newly blocked arm, and the arm directly opposite the home arm ( $\operatorname{arm} 5)$ was not used. There were a total of four novel configurations (1-3-6, 1-3-8, 1-2-7, and 1-4-7), each of which included the familiar home arm, a familiar outside arm, and a novel outside arm. The order of exposure to the novel configurations was counterbalanced across animals. A correct trial was defined as a trial in which the animal ran from the home arm to the correct outside arm and then back to the home arm. The animal was presented with each novel configuration until it reached $75 \%$ correct in the novel configuration or until it had $3 \mathrm{~d}$ of experience on that configuration. The animals were free to enter the arms in any order and spend as long in it as they desired. This experiment approximates natural exploration, because a foraging rat would normally explore outward from a familiar home nest (Barnett, 1975).

The recordings were performed using the Spike real-time linux data acquisition package [written by L. Frank, M. Okatan (Massachusetts General Hospital, Boston, MA), and P. Alvarez (Neural Arts, Boston, MA)]. Unit data were collected from every tetrode and filtered between either $300 \mathrm{~Hz}$ and $6 \mathrm{kHz}$ or $600 \mathrm{~Hz}$ and $6 \mathrm{kHz}$. Because the cortical electrodes were adjusted each day, there was some slight movement of the CA1 electrodes as well, so it was generally not possible to track individual CA1 neurons across days. We therefore treat each day of recording as a separate data set. Local field potential (LFP) data were recorded from one channel of each tetrode and from two to four dedicated LFP tetrodes. We selected an LFP electrode located in the deep layers of the entorhinal cortex for the theta analyses (see below), because theta is very clearly visible in this region. Images from an overhead camera recorded the behavior, including the location of a large front and small rear cluster of infrared diodes, and these images were compressed and saved to disk at $30 \mathrm{~Hz}$. After data acquisition, the animal's position was reconstructed from the stored images. Because the entire video record was stored, we were able to fill in the positions of occluded diodes using an interactive display program.

Data analysis. Clustering of the data into single units was performed using the XClust package (written by M. Wilson, Massachusetts Institute of Technology, Cambridge, MA). Great care was taken to only cluster well isolated neurons with spike waveform amplitudes that were clearly stable over the course of during session, because clustering errors could lead to artifacts in the plasticity analyses. All subsequent analyses were performed using custom written scripts and $\mathrm{C}$ programs running in Matlab (Mathworks, Natick, MA).

Many of the analytical techniques have been described previously (Frank et al., 2000, 2002), and only those that differ substantively from those studies are described here. We converted positions into distances from the end of the home arm and filtered the data by removing samples associated with low velocity $(<3.5 \mathrm{~cm} / \mathrm{sec})$ and low theta amplitude rhythm. That filtering removed intervals of inactivity when place-specific firing is typically not present (Buzsaki et al., 1983). Theta was extracted by bandpass filtering the LFP signal from the theta electrode between 6 and $14 \mathrm{~Hz}$. The theta signal was squared and then smoothed with a 2 -sec-wide Gaussian with a SD of $0.25 \mathrm{sec}$. A threshold determined by the experimenter was used to define low theta amplitude intervals. The velocity was computed as described previously (Frank et al., 2000).

To examine the moment-by-moment changes in place field structure, we applied an adaptive estimation algorithm that allowed us to characterize both the instantaneous place field structure (spatial intensity function) as well as the instantaneous interspike interval structure (temporal intensity function) of the spike train (Frank et al., 2002). We separated runs in each configuration into four "trajectories" from one arm to another: home to left, left to home, home to right, and right to home. We characterized the temporal intensity function with a single curve that was applied to all trajectories and estimated separate spatial intensity functions for each trajectory so that we could describe both the overall temporal structure of the spike train as well as the specific changes in spatial structure in each arm. Here, we focus on the changes in the spatial intensity function, however. The approach used to estimate the intensity functions was the same as that used in the previous study, with two exceptions. First, because the environment in this study was smaller than that of the previous study, the spatial control points were spaced $5 \mathrm{~cm}$ apart rather than 10. The spacing of the control points determines the minimum feature size that can be represented, and the spacing we chose allowed us to capture spatial features of place fields as small as $3 \mathrm{~cm}$, because an inspection of the firing rate function suggested that this feature size was more reasonable for the smaller environment.

Second, to determine the initial state of the spatial and temporal intensity functions, we simplified our previous procedure and ran the algorithm forward and backward in time to alternately update the spatial and temporal components of the firing rate until they converged. We previously found that this forward-backward approach resulted in accurate estimates of both the initial state of the system as well as its evolution over time. Preliminary analyses indicated that many neurons showed very fast spatial changes but relatively slower temporal changes. As such, we chose a spatial learning rate of 3.0 and a temporal rate of 0.05 based on previous simulation studies (Frank et al., 2002). We also ran the analyses with a variety of other learning rates to ensure that our results were not dependent on a particular choice of parameters. Only neurons with an average integrated spatial intensity (spatial area) of 100 or above were included in this study because that level corresponded to neurons with clear 
place fields. As in our previous study, the algorithm was run with a time step of $2 \mathrm{msec}$.

CA1 place fields show directional tuning in linear environments (McNaughton et al., 1983). We therefore separated outbound (home arm to an outside arm) and inbound (outside arm to home arm) runs based on the animals' head direction. In some trials, animals backed out of the outside arms and turned in the center of the maze rather than turning at the end of the outside arm so that the head direction was opposite the direction of motion. Because the difference between the effect of movement direction and head direction on hippocampal place fields is not well understood, we repeated all of our analyses using movement direction to define inbound and outbound runs. Our findings agreed closely for the two cases, so we present only the analyses organized by head direction.

We separated cells into putative excitatory and putative fast-spiking (FS) inhibitory groupsbased on spike waveform shape and firing rate (Fox and Ranck, 1981; Frank et al., 2001) and analyzed these cell types separately. We examined several measures of place field structure, including area, scale (SD), mean, median, interquartile range, and skewness. We found that although many of these measures showed patterns of change, place field area, measured as the area of the spatial intensity function, provided the most interpretable quantification of place field plasticity. To analyze the overall change in place cell activity, we calculated the proportional change of each place field as the integrated spatial intensity (spatial area) of the place field at the end of the last pass through the novel arm divided by the integrated spatial intensity at the end of the first pass. We identified rapidly changing place fields as those fields that changed in proportional area by a factor of three or more over the course of the first day of exposure to the novel environment.

We analyzed the time course of plasticity by performing an optimal polynomial fit to the curve describing the normalized area of the spatial intensity function as a function of time spent in the arm. The area was normalized by its mean so that cells with different levels of activity could be compared. The degree of this polynomial was constrained to be between 1 and either one-fourth the number of data points or 6 , whichever was smaller. This resulted in relatively smooth curves. The fitting procedure estimated the minimum degree of the polynomial that fit the data using Aikaike's information criterion. The resulting polynomials effectively characterized the shapes of the area curves without evident overfitting. In particular, the polynomials tended to underestimate rather than overestimate the rates of change, and thus they provided a conservative estimate of the dynamics of place fields. Examples of these fits are shown in supplemental material (available at www.jneurosci.org)

We then sampled the derivative of the polynomial every $5 \mathrm{sec}$ as an estimate of the rate of change of the spatial intensity function. We chose a sampling rate of once every $5 \mathrm{sec}$ because it represented a relatively long interval between samples. The longer the interval, the fewer data points each cell contributes, and the $5 \mathrm{sec}$ samples represent a conservative sampling interval that nevertheless allowed us to accurately describe the patterns of change in the data. We examined the distributions of derivatives as a function of the total number of minutes of experience across days or the number of minutes of experience within $1 \mathrm{~d}$.

For data from a given minute of experience to be included in the analyses, it had to meet three criteria: (1) it must contain data from at least two different animals; (2) it must include data from at least three different data sets; and (3) it must include data from at least 10 place fields. For simplicity, we present the day in terms of minutes of experience, but the results were consistent across temporal bin sizes from $30 \mathrm{sec}$ to $2 \mathrm{~min}$. When we analyzed the data as a function of the total amount of experience, data
Figure 1. A, The familiar and novel configurations of the T-maze task. In the familiar configuration, the animals ran between been visited previously (e.g., arm 6). B, Correlations of place field structure from run 1 to run 2 across the $3 \mathrm{~d}$ of exposure tc and the adjacent familiar arm in run 1, whereas the red bar represents the correlations for the familiar outside arm that was visite in both runs 1 and 2 . The asterisks denote significant differences $(p<0.00001)$. C, The place-related activity of a neuron that was active in the familiar configuration and maintained the same place field in the novel configuration. The top row shows the color-coded firing rate on the four trajectories through the familiar environment (depicted below each plot). The firing rate color map is shown to the right of the plots. The bottom row shows the firing rate in the novel environment. $D$, The place-related activity of a neuron recorded simultaneously from the same tetrode that was not active in the familiar configuration but developed a place field in the novel arm. The trajectories are the same as in C. 
were included in a given minute if there were at least $45 \mathrm{sec}$ spent in the arm during the minute of experience. Thus, if an animal had $1 \mathrm{~min}$ and $10 \mathrm{sec}$ of experience in the novel arm on day 1 , we included the data from first minute of experience on day 1 in the $0-1 \mathrm{~min}$ bin, whereas the data from the first $50 \mathrm{sec}$ of experience on day 2 would be included in the 1-2 min bin.

We measured the average amount of plasticity during each minute by constructing a plasticity index. The indices for each minute were computed as the absolute values of the derivatives sampled during that minute. Higher plasticity indices correspond to larger changes in place field area.

We also analyzed the activity of FS putative inhibitory interneurons. For these neurons, we directly estimated the average spatial firing rate by setting the learning rate of the temporal intensity portion of the adaptive algorithm to 0 . Because the temporal intensity was initialized to 1 for all values, and because the firing rate is estimated as the product of the spatial and temporal components of the model, a learning rate of 0 effectively removes the temporal component, leaving only a spatial component that corresponds directly to firing rate. To compute the relative change in that rate over time, we normalized the activity of each neuron to its initial value and plotted the average normalized rate across both head directions as a function of time. We also computed a directionality index for each neuron in each arm as the absolute value of the difference in area for the two spatial intensity function (one for each direction) divided by the sum of the areas. That produced an index with values between 0 and 1. Finally, we computed the average velocity during each minute of experience within $1 \mathrm{~d}$. The velocities were computed using all data that were not excluded by the velocity/theta exclusion described above. This analysis therefore included all points in which the animal was actively exploring. We then calculated the mean and SE of the velocity during each minute of experience, including only those minutes with velocities from at least three different data sets.

\section{Results}

We focus first on the putative excitatory neurons. These analyses reflect the activity of 240 neurons in the familiar configuration, 104 neurons in the first novel exposure (day 1), 89 neurons in the second novel exposure (day 2), and 60 neurons in third novel exposure (day 3). These neurons were recorded during 25 exposures to the familiar configuration, and 14, 13, and 7 first, second, and third exposures, respectively, to the novel configuration. The smaller number of third exposures resulted from the criterion used to determine when animals had learned the task in each novel configuration (see Materials and Methods). The total numbers of neurons active in the novel configurations were as follows: animal 1, 70; animal 2, 104; animal 3, 43; animal 4, 36.

\section{Place cell numbers and place field shapes}

To determine whether hippocampal activity was different in the novel and familiar locations, we examined the number of place fields and the patterns of place cell activity in the familiar outside and novel arms. We found that on day 1 and day 2 there were significantly more place fields in the novel arm compared with the familiar arm, whereas on day 3 the number of place fields did not differ between the arms. On day 1, of 104 active neurons, 52 had a place field in the novel arm, whereas 33 had a place field in the familiar arm $(p<0.01 ; Z$ test $)$. Similarly, on day 2 , of 88 active neurons, 42 had a place field in the novel arm, whereas 26 had a place field on the familiar $\operatorname{arm}(p<0.01 ; Z$ test). On day 3 , however, of 60 neurons, 26 had a place field on the novel arm, whereas 24 had a place field on the familiar $\operatorname{arm}(p>0.2 ; Z$ test $)$.

Our analysis of patterns of place field activity revealed that place cell activity was different between the novel and familiar locations. For each cell, we computed the correlation between the average firing rate profile in the first and second runs (Fig. $1 B$ ). For the novel arm (e.g., arm 6, run 2), we used the profile of the
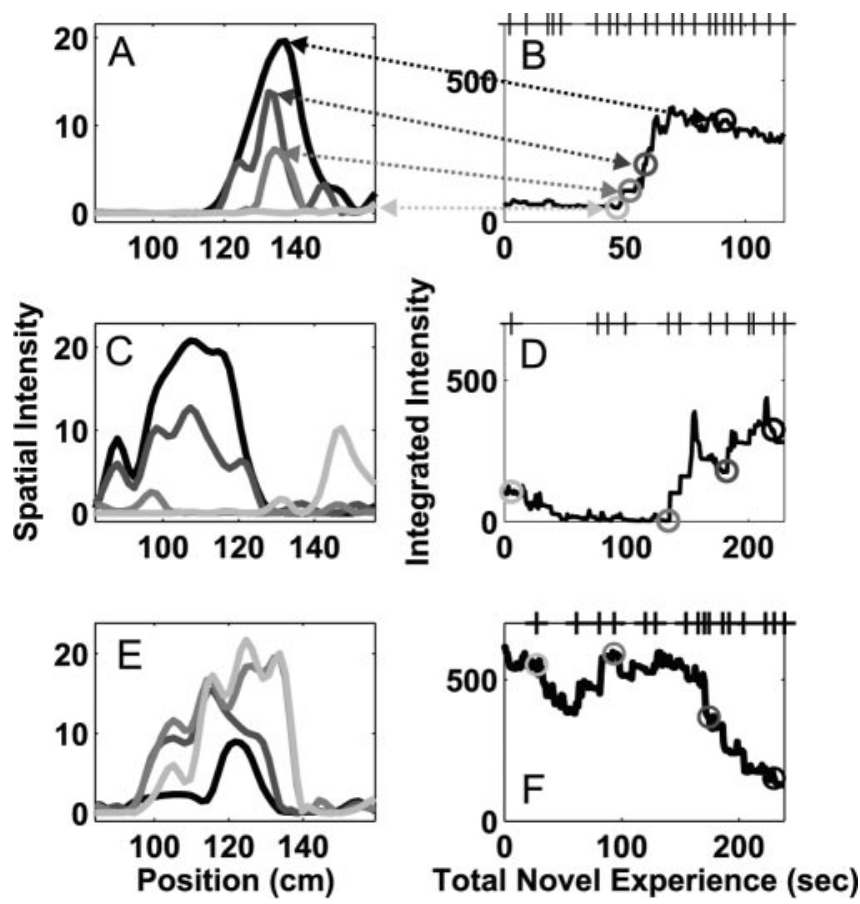

Figure 2. Place field plasticity on the first day of exposure to the novel arm. Each row represents the activity of a single neuron in one direction on the track. $A, C, E$, Examples of the spatial intensity (place field) at four different times. $B, D, F$, The integrated spatial intensity plotted as a function of the total experience in the novel arm. The four gray circles represent the times for the place fields shown in $A, C$, and $E$. The tick marks on the top $x$-axis represent the times at which the animal completed a full pass through the novel arm. These three neurons illustrate the variety of changes seen during the novel experience, including the sudden development of new fields $(A, B)$, changes in location of fields $(C, D)$, and decreases in activity of neurons with fields that were apparent from the beginning of the experience $(E, F)$.

corresponding familiar arm (e.g., arm 7, run 1) from the previous familiar configuration. The average correlations for the familiarfamiliar comparison were high across all $3 \mathrm{~d}$, indicating that place cells had similar fields on the familiar arm of runs 1 and 2 (Fig. $1 C)$. In contrast, the average correlations for the novel-familiar comparison were small on all $3 \mathrm{~d}$ (Fig. $1 D$ ) and were significantly smaller than the average correlation for the familiar-familiar comparison on all $3 \mathrm{~d}$ ( $p<0.00001$; Wilcoxon rank-sum test). The novel arm correlation was significantly greater than zero on day 1 ( $p<0.005$; $t$ test), but it declined and was not different from zero on days 2 and 3 ( $p>0.1 ; t$ test). Thus, both the number of active neurons and the structure of individual place fields strongly differentiated between the novel and familiar locations.

\section{Place field dynamics across multiple days}

To characterize the time course of place field formation, we examined in detail the patterns of change in CA1 place fields across the $3 \mathrm{~d}$ of exposure to the novel arm. We found that there were large changes in place field structure on days 1 and 2 but that by day 3 place fields had become stable. We looked at the dynamics of the place field (spatial intensity function) using our point process adaptive estimation algorithm (Brown et al., 2001; Frank et al., 2002).

The place field dynamics on day 1 included both the formation of new place fields (Fig. $2 A, B$ ) as well as changes in fields already present on the first pass through the new arm (Fig. $2 C-F$ ). Because hippocampal neurons often fire directionally in linear environments, we analyzed inbound and outbound runs separately (see Materials and Methods). We found that of 87 place fields from 50 cells active in the novel arm on day 1, 60\% (52 

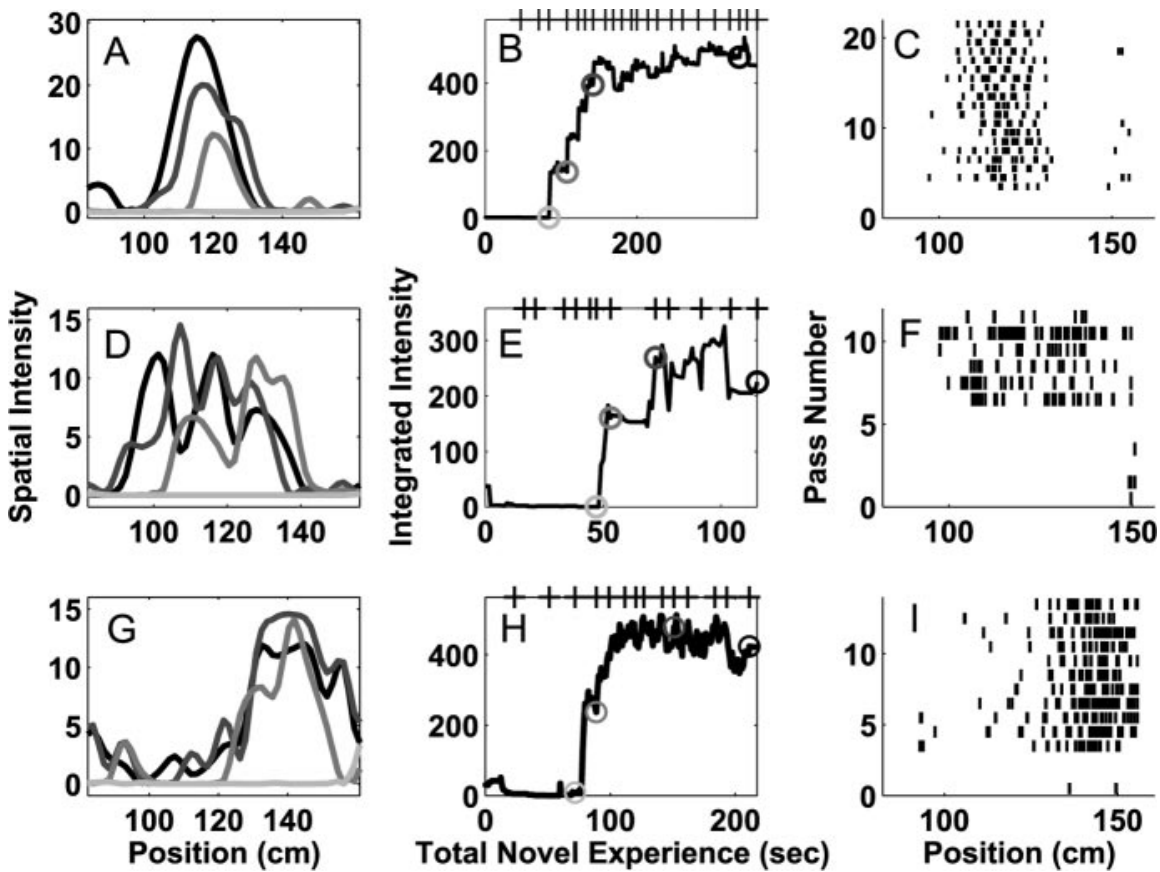

Figure 3. Examples of rapid place field formation in three neurons during the first day of exposure to the novel arm. Each row represents the activity of a single neuron in one direction on the track. As in Figure $2, A, D$, and $G$ represent the place field at four times corresponding to the gray circles in $B, E$, and $H$, and the tick marks on the top $x$-axis represent times when the animal completed a full pass through the novel arm. $C, F, I$, The spike rasters showing the activity of the neuron on each pass through the field. These neurons showed little or no activity for the first 1-2 min of experience in the novel arm, followed by the rapid emergence of place-specific firing. Once place-specific activity was visible, it evolved over the course of the session with a declining rate. These plots show that the animal traversed the entire arm three to six times before the place field became visible and that the place fields developed rapidly over the course of one to two passes. These raster plots must be interpreted cautiously because they do not include information about the amount of time the animal spent in each location.

fields, 36 cells) showed an increase in activity, whereas 40\%(35 fields, 26 cells) showed a decrease. In most cases ( 74 fields, 37 cells), a single cell had a place field on the novel arm in each of the two directions of motion, and these fields could show either the same ( 50 fields, 25 cells) or opposite ( 24 fields, 12 cells) directions of change. The magnitude and direction of these changes varied among simultaneously recorded neurons.

On the first day of exposure, many place fields (23 of 87) developed very rapidly (Fig. $3 A, B, D, E, G, H$ ). Place-specific activity in these neurons was often undetectable for the first 1-2 min of experience in the novel arm, although the animals made as many as six passes through the arm during that time. After that initial period of inactivity, place fields became visible in as few as $5-15 \mathrm{sec}$ (one to two passes). In several instances, place fields appeared in locations in which no previous spiking had been observed (Fig. $3 C, F, H$ ). The developmental time course of one of these fields is shown in the supplemental material (available at www.jneurosci.org).

We further found that place field plasticity on day 2 depended critically on the length of experience on day 1 (Fig. 4). We computed the derivatives of the normalized place field area in the novel arm and examined the cumulative distribution of these derivatives as a function of the total time of experience, the total number of complete passes, and the number of days of exposure. We found that when the animal had spent $<4$ min in the novel arm on day 1, the cumulative distributions of derivatives were wide, indicating that place fields changed substantially on day 2 . In contrast, when the animal had spent $>5 \mathrm{~min}$ in the novel arm on day 1 , the cumulative distributions were much narrower, indicating that place fields changed much less on day 2 (Fig. 5A). Thus, place fields became stable after $\sim 5-6 \mathrm{~min}$ of experience in the novel arm. These patterns were not clearly evident when the data were organized according to the number of passes through the arm (Fig. 5B). The differences in the amount of change as a function of time as opposed to passes resulted from large variability in the length of time the animals spent traversing the novel arm during exploration.

Our results also indicated that short exposures on day 1 led to "priming" of plasticity on day 2. That is, when the animal had $<3$ min of experience on day 1 , then the third minute of experience fell on day 2 , and the plasticity indices for that minute were significantly larger than the indices at every other minute of experience in the novel arm (Fig. 5C) $(p<0.00001$; Wilcoxon rank-sum tests). The same pattern could be seen when the data were organized by total experience, but only the first minute of experience on a given day was included in the analyses (data not shown). These results were not visible when the data were organized by the number of minutes of experience within $1 \mathrm{~d}$ (Fig. 5D).

The plasticity indices measured on day 3 ( $\min 7$ and 8 ) were small and were not different from the corresponding indices from day 2 (Fig. 5C). In addition, when the data were organized by experience within $1 \mathrm{~d}$ (Fig. 5D), the plasticity indices for the first $2 \mathrm{~min}$ of day 3 were lower than those of days 1 and 2 ( $p<0.01$; post hoc tests; Kruskal-Wallis ANOVA). In summary, the rapid changes seen on day 1 and the priming seen on day 2 were not present on day 3 , indicating that that hippocampal representation of space had stabilized by the third day of exposure to the novel place.

\section{Comparison of place field properties and dynamics}

On each day, the animal experienced both familiar and novel arms, therefore we compared place field properties and dynamics in the novel arm with those in the familiar arm. We first examined place field directionality (Fig. 6A) and found that it differed between the novel and familiar arms on days 1 and 2 (two-way ANOVA; Tukey-Kramer post hoc tests; $p<0.05$ for days 1 and 2). There was also a trend for place cells to be more directional on the novel arm on day 3, but it did not reach the criterion level of significance $(p<0.08)$. In addition, directionality for day 3 in the novel arm was significantly higher than directionality on day 1 in the novel arm $(p<0.05)$, but there were no significant differences among the directionality indices for the familiar arm. Thus, place fields became more directional in the novel arm over the $3 \mathrm{~d}$ of experience and approached the level of directionality seen in the familiar arm.

We also found that place field changes in the novel arm were larger on days 1 and 2 compared with those in the familiar arm, but that on day 3 place field changes in the novel and familiar arms were similar. The proportional change in the novel arm on day 1 (Fig. 6D) and day 2 (data not shown) was significantly larger that that seen in the familiar arms of both the novel and familiar configurations (Fig. $6 E, F)(n>35$ fields; $p<0.01$; Wilcoxon rank-sum tests). In contrast, the proportional change 

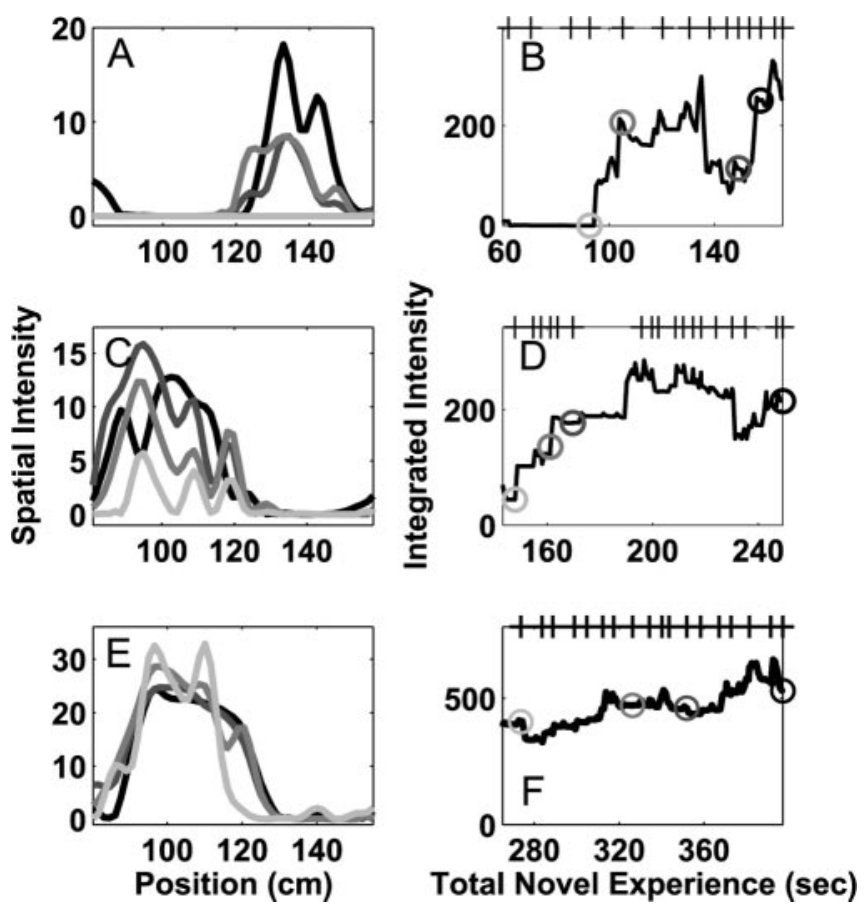

Figure 4. Place field plasticity during the second day of experience. As in Figure $2, A, C$, and $E$ represent the place fields of three neurons at four times corresponding to the gray circles in $B$, $D$, and $F$, and the tick marks on the top $x$-axis represent at which the animal completed a full pass through the novel arm. The integrated intensity is plotted as a function of the total novel experience, computed as the length of experience on day 1 plus the length on day 2 . In most cases in which the animal had little experience during the first day, cells showed large, fast changes on day 2 that were qualitatively similar to the fastest changes seen during day 1 . In contrast, as the amount of experience increased, the amount of place field plasticity decreased $(C-F)$.
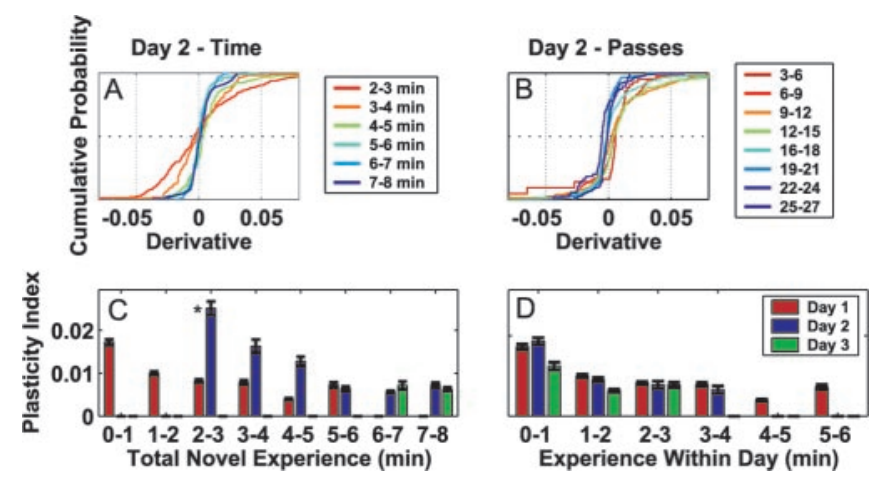

Figure 5. Place field plasticity over days. $A$, The cumulative distribution of the derivatives of the place field area as a function of the total experience in the novel arm on day 2. Each color corresponds to a particular number of minutes of experience (see legend). $B$, The cumulative distributions of derivatives on day 2 as a function of the total number of complete passes through the place. $C$, The plasticity indices as a function of total experience for each minute on each day. The index was calculated as the mean absolute value of the derivatives from each minute and measures the overall tendency to show changes. Bars represent mean $\pm S E M$. $D$, The plasticity indices as a function of experience within each day. The missing bars in $C$ and $D$ represent minutes of experience that did not contain sufficient data sets or numbers of neurons to be included in the analyses.

on day 3 of the novel exposure did not differ from that seen in the familiar arms ( $p>0.1$; Wilcoxon rank-sum test). We also found that the priming of plasticity on day 2 associated with short experiences on day 1 was not present in the familiar environment. The mean plasticity index for the third minute of experience in the familiar arm was the same whether that minute occurred on the first or second day of exposure (Fig. $6 B)(p>0.5$; Wilcoxon rank-sum test), and the plasticity indices for the first minute on each day were also similar (Fig. 6C). Thus, although novel and familiar arms showed different patterns of change for the first $2 \mathrm{~d}$ of exposure, the patterns were the same on the third day.

\section{Plasticity of inhibitory activity}

To determine whether the changes in place fields reflect changes across the hippocampal network, we analyzed the firing of simultaneously recorded FS putative inhibitory neurons in the novel and familiar arms (day 1, $n=14$; day 2, $n=10$; day 3, $n=6$ ). We noted two characteristics of these neurons. First, during the first minute of experience on day 1, FS neurons were less active in the novel arm compared with the familiar arm (Table 1) $(p<0.05$; Tukey-Kramer post hoc test), but the firing rates did not differ for subsequent minutes $(p>0.1)$.

Second, the dynamics of inhibitory firing rates also changed across the $3 \mathrm{~d}$ of exposure. Of the 28 inhibitory fields ( 14 neurons, two head directions) recorded in the novel arm on the first day, 21 increased in firing rate as measured by a linear regression of mean rate versus time. Overall, that corresponded to an increase in inhibitory activity of $20 \%$ over 2 min of experience on day 1 (Fig. 7A). In contrast, inhibitory activity decreased in the familiar arms across all $3 \mathrm{~d}$ of exposure and in the familiar configuration (increasing fields: familiar arm, novel configuration: day 1, 7 of 28; day 2, 3 of 20; day 3, 1 of 12 ) (Fig. 7D-G). The mean firing rate was approximately constant on the second day in the novel arm in which 9 of 20 inhibitory fields increased in rate (Fig. 7B). Only on the third day did inhibitory neurons in the novel arm show the same decrease in rate seen in the familiar arms in which 12 of 12 fields showed a decrease in rate (Fig. 7C). Thus, only on the third day of exposure to the novel place did excitatory and inhibitory neurons show the same patterns of change in the novel and familiar locations.

\section{Behavioral change}

To understand the relationship between the time course of neural change and the time course of behavioral change, we analyzed the animals' behavior in the novel and familiar arms. We chose running velocity as a behavioral measure of familiarity because animals exploring a novel environment initially move slowly and engage in substantial exploration, whereas animals run rapidly to and from food wells in familiar environments. We found that the animal's behavior remained different in the novel arm across all $3 \mathrm{~d}$ of exposure (Fig. 8). We first examined the velocities in the novel arm. We found that animals ran more slowly during the first day of exposure to the novel arm than during the second day of exposure ( $p<0.001$; two-way ANOVA with Tukey-Kramer post hoc tests). In addition, novel arm velocity was approximately constant on the first day but showed a clear decreasing trend on the second day $(p<0.001)$. There were no differences between the velocities in the novel arm on day 2 versus day 3 .

We then compared the velocities in the novel and familiar arms. The velocity in the novel arm was significantly lower than the velocity in the familiar arm on each of the $3 \mathrm{~d}$ of exposure $(p<0.001)$. The velocities were also lower on all $3 \mathrm{~d}$ when examined as a function of the total experience (data not shown). A similar pattern was seen in the total amount of time spent in each arm. For each novel exposure, we computed the difference between the amount of time the animal spent in the novel versus the familiar outside arm. Animals spent, on average, $68 \mathrm{sec}$ more in the novel arm on day 1, $37 \mathrm{sec}$ more in the novel arm on day 2, and $43 \mathrm{sec}$ more on day 3 . These differences were significant $(p<$ 

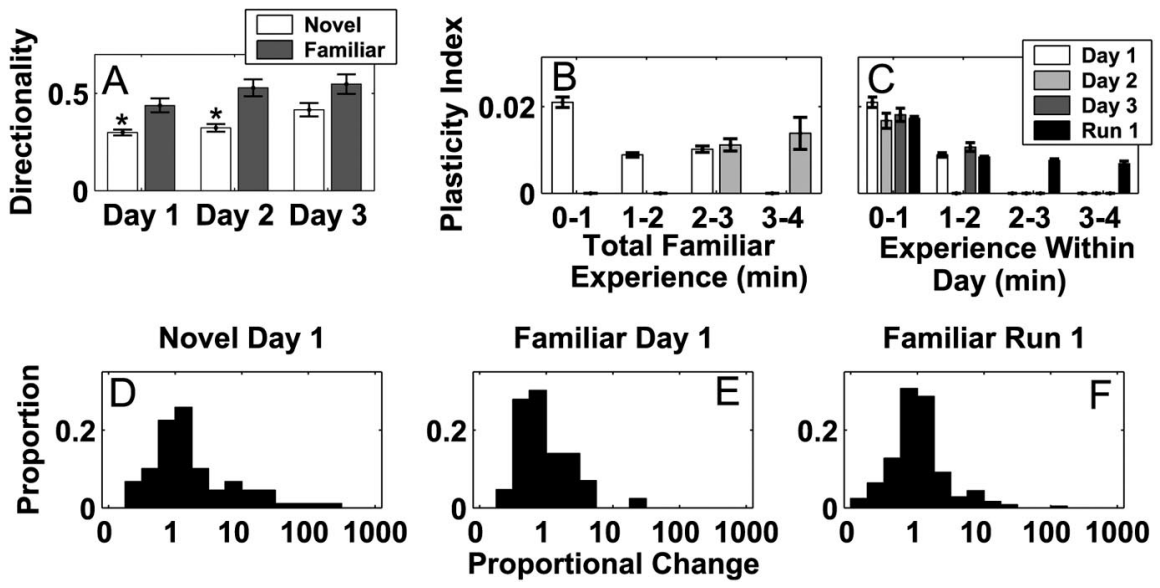

Figure 6. Place field properties in the novel and familiar environments. $A$, Place field directionality in the novel and familiar arms across the $3 \mathrm{~d}$ of experience. The asterisks represent a significant difference within the day. $B$, The plasticity indices for place fields in the familiar arm as a function of total experience in the familiar arm for each minute on days 1 and 2 . There were insufficient data to perform the same analysis on day 3 . The increase in plasticity seen in the novel arms was not present in the familiar arms. C, The plasticity indices as a function of experience within $1 \mathrm{~d}$ for the familiar arm days $1-3$ and for the familiar arms in the run 1 familiar configuration. $D-F$, Histograms of the proportional change of place fields recorded from neurons active in the novel arm on day $1(A)$, the familiar arm on day $1(B)$, or in one of the familiar arms from the run 1 in the familiar configuration $(C)$. The proportional change was, on average, larger in the novel arms.

Table 1. Firing rates of inhibitory neurons on day 1

\begin{tabular}{llll}
\hline & \multicolumn{3}{l}{ Experience within day 1 (minutes) } \\
\cline { 2 - 4 } & $0-1$ & $1-2$ & $2-3$ \\
\hline Novel rate $(\mathrm{Hz})$ & $31.5 \pm 2.4$ & $31.0 \pm 3.1$ & $38.9 \pm 3.6$ \\
Familiar rate $(\mathrm{Hz})$ & $39.9 \pm 1.9$ & $35.1 \pm 1.9$ & $35.9 \pm 3.2$ \\
\hline
\end{tabular}

Each value represents mean $\pm S E M$. The firing rates in the novel and familiar environments differed during the first minute but not during subsequent minutes.

0.03; paired $t$ tests) but did not differ among the $3 \mathrm{~d}$. Hence, although there were some indications that the animals' behavior in the novel arm changed from the first to the second day, it remained different from that seen in the familiar arm even on the third day of exposure.

\section{Discussion}

The hippocampus is essential for spatial learning, but very little is known about the changes in neural activity that occur as a new spatial representation forms. We studied changes in hippocampal neural activity and changes in behavior across $3 \mathrm{~d}$ of exposure to a set of novel locations in an effort to identify the changes in neural

activity that occur as animals learn about new places. Previous examinations of place cell plasticity in novel environments relied on averaging data from many neurons across wide time bins ( $\geq 3$ min) (Bostock et al., 1991; Wilson and McNaughton, 1993; Nakazawa et al., 2003). Examining the place-specific firing of single neurons at shorter time scales is hampered by the stochastic nature of neural firing: although it is possible to produce estimates of place fields from shorter (e.g., $1 \mathrm{~min}$ ) sections of data (Lever et al., 2002), accurately quantifying place cell properties using standard averaging methods at that time scale is difficult, if not impossible. Because our methods allowed us to examine the changes in neural receptive fields on a millisecond time scale (Frank et al., 2002; Eden et al., 2004), they permitted both a more accurate description of neural plasticity as well as direct comparisons of the rates of neural change to the rates of behavioral change and, in principle, to the rates of the cellular and molecular processes underlying plasticity.
When we examined the formation of place representations in the novel arm, we found that although most neurons were active during the first traversal of the new place, many previously silent neurons developed place specific activity as the animal explored the novel arm. These findings extend previous observations from Hill (1978), who described 2 of 12 place cells as initially silent in a novel environment. We found place-specific activity could emerge in a particular neuron over as little as $5 \mathrm{sec}$ comprising a single pass, even if there had been no spiking on previous passes. These findings are consistent with results from a location-scene learning task in monkeys in which it was observed that hippocampal neurons show rapid changes during learning (Wirth et al., 2003).

Place field change in familiar environments has previously been hypothesized to depend on spike timing-dependent plasticity (STDP) in which presynaptic activity must precede postsynaptic spiking to cause an increase in synaptic efficacy (Abbott and Blum, 1996; Blum and Abbott, 1996; Mehta et al., 1997, 2000; Bi and Poo, 1998; Ekstrom et al., 2001). The rapid emergence of place fields without previous activity cannot be accounted for by STDP in CA1, however, because there was often no postsynaptic spiking observed before the development of the place field. An alternate possibility is that these changes result from a decrease in inhibition that allowed previously subthreshold activity to become suprathreshold. We think that unlikely; although most interneurons were initially suppressed in the novel arm, their firing rates quickly recovered to levels similar to those found in the familiar arm. Thus, inhibitory activity was increasing as the rapid development of place fields occurred. Nonetheless, it is possible that the rapid emergence of place fields occurred in those excitatory neurons where activity was influenced primarily by inhibitory neurons with decreasing firing rates. These changes could also result from plasticity that is independent of postsynaptic spiking (e.g., dendritic spikes) (Golding et al., 2002) or reorganizations of the entorhinal cortical and CA3 inputs to CA1 (Amaral and Witter, 1995). Additional work will be necessary to determine the exact mechanism.

Previous work has shown that reconstructions of the animal's position in a novel open field environment were more accurate when the second $10 \mathrm{~min}$ of data were used compared with the first 10 min (Wilson and McNaughton, 1993). That finding is consistent with our results, but because the exact time scale of these changes and the stability of fields across multiple days was not examined, a direct comparison of the two studies is difficult. Our findings for changes in interneuron rates are also consistent with previous work. Wilson and McNaughton (1993) reported that 10 of 15 interneurons decreased their rates in a novel open field environment, whereas Fyhn et al. (2002) reported that alterations in the location of the escape platform in a familiar water maze caused a minority of inhibitory interneurons to temporarily decrease their rates. Our work extends these findings because we determined that interneuron rates, although initially significantly smaller in the novel arm, rose to comparable levels after the first minute of experience. We hypothesize that the initial decrease in 
inhibitory activity may facilitate plasticity in CA1 and that, because CA1 neurons develop fields, the strong connections between excitatory and inhibitory neurons (Csicsvari et al., 1999; Frank et al., 2001) may drive the simultaneous increase in inhibitory activity.

We observed substantial place field plasticity during the first minute of experience on day 1 , but the largest overall changes in neural activity occurred on day 2 when the animal was reexposed to an environment that had been experienced for $<3 \mathrm{~min}$ on day 1 . Thus, our results indicate that 1-2 min of experience on the first day were not sufficient for the hippocampus to form a stable and enduring representation. In contrast, the numbers of active neurons, the directionality of place fields, the plasticity indices, and the firing rates of inhibitory interneurons all support the claim that place fields were stable on the third day once the animal had at least 5-6 min of experience. These findings suggest that the amount of time spent in a location is the critical determinant of the stability of the place representation.

Current theories suggest that the patterns of activity seen in the hippocampus during awake immobility and sleep are important in memory consolidation in which initially labile representations are converted into a more long-lasting form (Wilson and McNaughton, 1994; Buzsaki, 1998; Dudai, 2004). Our data indicate that this conversion did not occur after short exposures to the novel place on day 1 but did occur after longer exposures, suggesting that there is a critical minimum length of experience required to trigger consolidation and produce a lasting hippocampal representation.

Our results also showed that change in plasticity on day 2 was more strongly related to length of time spent in the novel arm rather than the number of passes through the novel arm. Previous analyses of place field plasticity in familiar environments organized the data as a function of passes through the field rather than time spent in the field (Shen et al., 1997; Mehta et al., 1997, 2000; Ekstrom et al., 2001; Frank et al., 2002). Because these environments were familiar, and because animals tend to run smoothly through familiar environments, it is likely that the number of passes was strongly correlated with the time spent in the place field in these studies. Nonetheless, our findings suggest that the actual length of experience is more closely related to place field plasticity than the number of individual experiences of the place. Taken together, these findings suggest that the length of experience, or some variable closely correlated with the length of experience, determines the stability of the cellular and molecular events underlying the development of spatial representations in the hippocampus require.

Although the stability of the hippocampal representation of space suggested that the novel arms were familiar by the third day of exposure, the animals continued to behave differently in the novel arm on the third day. The animals' velocities in the familiar arm showed the previously observed pattern in which running speed was initially high and then declined over the course of the
Day 1

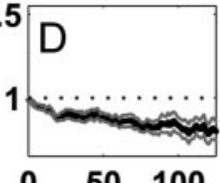

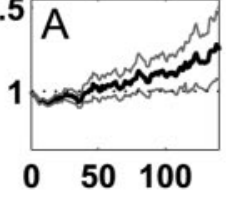

Day 2
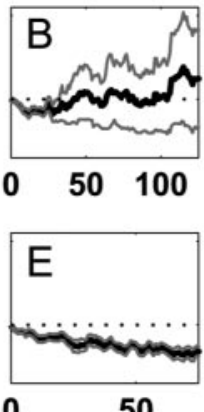

Experience Within Day (sec)
Day 3

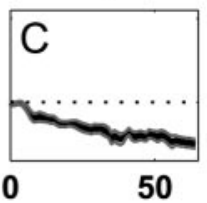

Run 1

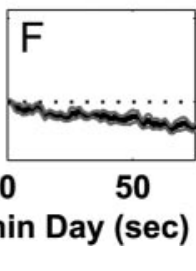

50

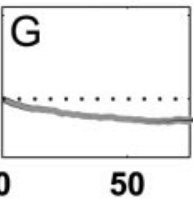

Figure 7. Changes in the activity of putative inhibitory interneurons in the novel and familiar arms. Each plot represents the average, normalized change in firing rate of all putative inhibitory neurons in the novel or familiar arm(s). $A-C$, The change in

Figure 8. The velocity of the animal in the novel and familiar arms on each day. Each plot shows the mean \pm SEM of the Day 2 Day 3 Run 1

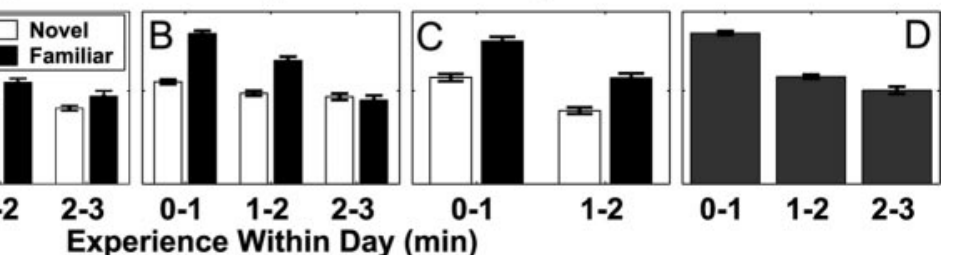
Experience Within Day (min)

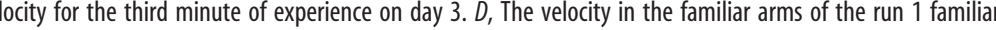
run (Mehta et al., 1997) and the velocities were very similar across the $3 \mathrm{~d}$ of exposure. Although that declining trend was visible on days 2 and 3 in the novel arm, the animals ran much more slowly in the novel arm compared with the familiar arm during the initial minute on all $3 \mathrm{~d}$. This occurred although other factors that are likely to influence velocity, such as motivation level and fatigue, were the same during those periods. Thus, even after $\geq 5$ min of experience in the novel arm across $3 \mathrm{~d}$, the animal did not treat the novel arm as a familiar location.

Stable hippocampal place fields are therefore not sufficient for an animal to treat a place as a familiar. We hypothesize that neocortical, and perhaps subcortical, regions outside the hippocampus continue to distinguish between the novel and familiar places even after the hippocampal representation has formed and hippocampal plasticity has declined. These results provide support for the hypothesis that the hippocampus is specialized for rapid learning, and that other brain regions form representations more slowly. Thus, our findings suggest that although the hippocampus may form new memories quickly, using those memories to guide behavior also requires changes in other brain regions.

\section{References}

Abbott LF, Blum KI (1996) Functional significance of long-term potentiation for sequence learning and prediction. Cereb Cortex 6:406-416.

Aggleton JP, Hunt PR, Rawlins JN (1986) The effects of hippocampal lesions upon spatial and non-spatial tests of working memory. Behav Brain Res 19:133-146.

Amaral DG, Witter MP (1995) Hippocampal formation. In: The rat nervous system (Paxinos G, ed), pp 443-493. San Diego: Academic. 
Barnett SA (1975) The rat. Chicago: University of Chicago.

Best PJ, White AM, Minai A (2001) Spatial processing in the brain: the activity of hippocampal place cells. Annu Rev Neurosci 24:459-486.

Bi GQ, Poo MM (1998) Synaptic modifications in cultured hippocampal neurons: dependence on spike timing, synaptic strength, and postsynaptic cell type. J Neurosci 18:10464-10472.

Blum KI, Abbott LF (1996) A model of spatial map formation in the hippocampus of the rat. Neural Comput 8:85-93.

Bostock E, Muller RU, Kubie JL (1991) Experience-dependent modifications of hippocampal place cell firing. Hippocampus 1:193-205.

Brown EN, Nguyen DP, Frank LM, Wilson MA, Solo V (2001) An analysis of neural receptive field dynamics by point process adaptive filtering. Proc Natl Acad Sci USA 98:12261-12266.

Buzsaki G (1998) Memory consolidation during sleep: a neurophysiological perspective. J Sleep Res 7 [Suppl 1]:17-23.

Buzsaki G, Leung LW, Vanderwolf CH (1983) Cellular bases of hippocampal EEG in the behaving rat. Brain Res 287:139-171.

Cassel JC, Cassel S, Galani R, Kelche C, Will B, Jarrard L (1998) Fimbriafornix vs selective hippocampal lesions in rats: effects on locomotor activity and spatial learning and memory. Neurobiol Learn Mem 69:22-45.

Csicsvari J, Hirase H, Czurko A, Mamiya A, Buzsaki G (1999) Oscillatory coupling of hippocampal pyramidal cells and interneurons in the behaving rat. J Neurosci 19:274-287.

Dudai Y (2004) The neurobiology of consolidations, or, how stable is the engram? Annu Rev Psychol 55:51-86.

Eden UT, Frank LM, Barbieri R, Solo V, Brown EN (2004) Dynamic analysis of neural encoding by point process adaptive filtering. Neural Comput 16:971-998.

Eichenbaum H, Cohen NJ (2001) From conditioning to conscious recollection. New York: Oxford UP.

Ekstrom AD, Meltzer J, McNaughton BL, Barnes CA (2001) NMDA receptor antagonism blocks experience-dependent expansion of hippocampal "place fields." Neuron 31:631-638.

Ekstrom AD, Kahana MJ, Caplan JB, Fields TA, Isham EA, Newman EL, Fried I (2003) Cellular networks underlying human spatial navigation. Nature 425:184-188.

Ferbinteanu J, Ray C, McDonald RJ (2003) Both dorsal and ventral hippocampus contribute to spatial learning in Long-Evans rats. Neurosci Lett 345:131-135.

Fox SE, Ranck JBJ (1981) Electrophysiological characteristics of hippocampal complex-spike cells and theta cells. Exp Brain Res 41:399-410.

Frank LM, Brown EN, Wilson MA (2000) Trajectory encoding in the hippocampus and entorhinal cortex. Neuron 27:169-178.

Frank LM, Brown EN, Wilson MA (2001) A comparison of the firing properties of putative excitatory and inhibitory neurons from CA1 and the entorhinal cortex. J Neurophysiol 86:2029-2040.

Frank LM, Eden UT, Solo V, Wilson MA, Brown EN (2002) Contrasting patterns of receptive field plasticity in the hippocampus and the entorhinal cortex: an adaptive filtering approach. J Neurosci 22:3817-3830.

Fyhn M, Molden S, Hollup S, Moser MB, Moser E (2002) Hippocampal neurons responding to first-time dislocation of a target object. Neuron 35:555-566.

Golding NL, Staff NP, Spruston N (2002) Dendritic spikes as a mechanism for cooperative long-term potentiation. Nature 418:326-331.

Hill AJ (1978) First occurrence of hippocampal spatial firing in a new environment. Exp Neurol 62:282-297.

Holscher C (2003) Time, space and hippocampal functions. Rev Neurosci 14:253-284

Hori E, Tabuchi E, Matsumura N, Tamura R, Eifuku S, Endo S, Nishijo H,
Ono T (2003) Representation of place by monkey hippocampal neurons in real and virtual translocation. Hippocampus 13:190-196.

Izquierdo I, Quillfeldt JA, Zanatta MS, Quevedo J, Schaeffer E, Schmitz PK, Medina JH (1997) Sequential role of hippocampus and amygdala, entorhinal cortex and parietal cortex in formation and retrieval of memory for inhibitory avoidance in rats. Eur J Neurosci 9:786-793.

Jarrard LE (1993) On the role of the hippocampus in learning and memory in the rat. Behav Neural Biol 60:9-26.

Knierim JJ (2002) Dynamic interactions between local surface cues, distal landmarks, and intrinsic circuitry in hippocampal place cells. J Neurosci 22:6254-6264.

Lever C, Wills T, Cacucci F, Burgess N, O’Keefe J (2002) Long-term plasticity in hippocampal place-cell representation of environmental geometry. Nature 416:90-94.

Markus EJ, Qin YL, Leonard B, Skaggs WE, McNaughton BL, Barnes CA (1995) Interactions between location and task affect the spatial and directional firing of hippocampal neurons. J Neurosci 15:7079-7094.

McNaughton BL, Barnes CA, O’Keefe J (1983) The contributions of position, direction, and velocity to single unit activity in the hippocampus of freely-moving rats. Exp Brain Res 52:41-49.

Mehta MR, Barnes CA, McNaughton BL (1997) Experience-dependent, asymmetric expansion of hippocampal place fields. Proc Natl Acad Sci USA 94:8918-8921.

Mehta MR, Quirk MC, Wilson MA (2000) Experience-dependent asymmetric shape of hippocampal receptive fields. Neuron 25:707-715.

Morris RG, Schenk F, Tweedie F, Jarrard LE (1990) Ibotenate lesions of hippocampus and/or subiculum: dissociating components of allocentric spatial learning. Eur J Neurosci 2:1016-1028.

Moser E, Moser MB, Andersen P (1993) Spatial learning impairment parallels the magnitude of dorsal hippocampal lesions, but is hardly present following ventral lesions. J Neurosci 13:3916-3925.

Muller R (1996) A quarter of a century of place cells. Neuron 17:813-822.

Nakazawa K, Sun LD, Quirk MC, Rondi-Reig L, Wilson MA, Tonegawa S (2003) Hippocampal CA3 NMDA receptors are crucial for memory acquisition of one-time experience. Neuron 38:305-315.

O’Keefe J, Dostrovsky J (1971) The hippocampus as a spatial map. Preliminary evidence from unit activity in the freely-moving rat. Brain Res 34:171-175.

O'Keefe J, Nadel L (1978) The hippocampus as a cognitive map. London: Oxford UP.

Pacteau C, Einon D, Sinden J (1989) Early rearing environment and dorsal hippocampal ibotenic acid lesions: long-term influences on spatial learning and alternation in the rat. Behav Brain Res 34:79-96.

Rawlins JN, Olton DS (1982) The septo-hippocampal system and cognitive mapping. Behav Brain Res 5:331-358.

Rolls ET (1999) Spatial view cells and the representation of place in the primate hippocampus. Hippocampus 9:467-480.

Shen J, Barnes CA, McNaughton BL, Skaggs WE, Weaver KL (1997) The effect of aging on experience-dependent plasticity of hippocampal place cells. J Neurosci 17:6769-6782.

Squire LR, Zola-Morgan S (1991) The medial temporal lobe memory system. Science 253:1380-1386.

Wilson MA, McNaughton BL (1993) Dynamics of the hippocampal ensemble code for space. [Erratum (1994) 264:16]. Science 261:1055-1058.

Wilson MA, McNaughton BL (1994) Reactivation of hippocampal ensemble memories during sleep. Science 265:676-679.

Wirth S, Yanike M, Frank LM, Smith AC, Brown EN, Suzuki WA (2003) Single neurons in the monkey hippocampus and learning of new associations. Science 300:1578-1581. 